\title{
A Highly Tunable Barium Strontium Titanate Thin Film MIM Varactor With Floating Metal
}

This paper was downloaded from TechRxiv (https://www.techrxiv.org).

LICENSE

CC BY-NC-SA 4.0

SUBMISSION DATE / POSTED DATE

$21-08-2021 / 25-08-2021$

CITATION

Nath, S (2021): A Highly Tunable Barium Strontium Titanate Thin Film MIM Varactor With Floating Metal. TechRxiv. Preprint. https://doi.org/10.36227/techrxiv.16048704.v1

$\mathrm{DOI}$

10.36227/techrxiv.16048704.v1 


\title{
A Highly Tunable Barium Strontium Titanate Thin Film MIM Varactor With Floating Metal
}

\author{
Surajit Kumar Nath, Graduate Student Member, IEEE, J. Pundareekam Goud, Sandeep Sharma \\ Kongbrailatpam, Guruswamy Rajaram, and K.C. James Raju, Senior Member, IEEE
}

\begin{abstract}
A ferroelectric metal insulator metal (MIM) varactor structure incorporating a floating metal and coplanar waveguide (CPW) has been introduced here. The work of the proposed varactor is based on the field-dependent material properties of $(\mathrm{Ba}, \mathrm{Sr}) \mathrm{TiO}_{3}(\mathrm{BST})$ thin film. A capacitance tunability of $44 \%$ has been achieved for the bias voltage of $0 \mathrm{~V}$ to $10 \mathrm{~V}$ over a frequency range of $1 \mathrm{GHz}$ to $3 \mathrm{GHz}$. The proposed varactor structure yields a compact area, high capacitance density, and reduced mask process ( 2 masks).
\end{abstract}

Index Terms - BST thin film, equivalent circuit, ferroelectric material, tunable microwave device, varactor.

\section{INTRODUCTION}

$\mathrm{V}$ OLTAGE-TUNABLE microwave devices are important for reconfigurable wireless systems such as cognitive radio, software-defined radio, and frequency-agile transceivers [1-3]. For an integrated solution, these voltage-tunable microwave devices must be compact and easy to manufacture. Ferroelectric materials show voltage-dependent material properties, such as tunable permittivity, and loss tangent, and are expected to be used in voltage-tunable microwave applications. Therefore, ferroelectric materials, particularly BST thin films have been extensively investigated in varactors $[4,5]$, delay lines [6], and filters $[7,8]$. In particular, the focus of this work is to develop a high-performance varactor incorporating the BST thin film. BST thin film-based varactor has advantages in terms of high tunability, high switching speed, high power handling capability, low operating voltage, and low packaging cost over its counterparts such as semiconductor diodes and MEMS devices [9]. This work employs $\mathrm{Ba}_{0.5} \mathrm{Sr}_{0.5} \mathrm{TiO}_{3}$ or BST5 thin film to develop the varactors because BST5 thin film offers high permittivity, high tunability, and high commutation quality factor $(\mathrm{CQF})$ or low loss at room temperature. However, the performance of the BST thin film varactor depends not only on the quality of the BST thin film, but also on the type of varactor structure used. Therefore it is important to find a compact varactor structure that has fewer process steps and simultaneously provides high capacitance tunability and quality

This work was financially supported by the Ministry of Human Resource Development (MHRD), India under grant UoH-IoE F11/9/2019-U3(A) and Department of Science \& Technology (DST), India under grant CRG/2019/000427 dated 27.01.2020. (Corresponding author: K.C. James Raju.)

Surajit Kumar Nath was with CASEST, School of Physics, University of Hyderabad, Hyderabad-500046, India. He is now with International College of Semiconductor Technology, National Yang Ming Chiao Tung University, Hsinchu-300, Taiwan. (email: skn.st06@nycu.edu.tw).

J. Pundareekam Goud, Sandeep Sharma Kongbrailatpam, Guruswamy Rajaram, and K.C. James Raju are with CASEST, School of Physics, University of Hyderabad, Hyderabad-500046, India. (email: kcjrsp@uohyd.ernet.in).

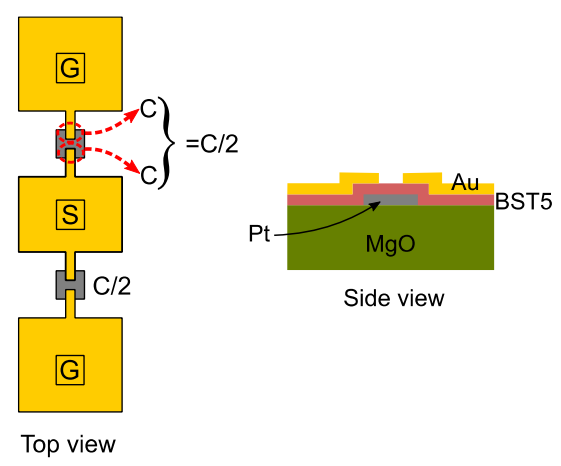

Fig. 1. Proposed BST thin film MIM varactor with floating metal.

factor (Q). Popular varactor structures are interdigitated capacitor (IDC) [10-12], MIM varactor [7], and circular patch capacitor (CPC) [13]. Due to the fringe field between the fingers, IDC has a large static capacitance and shows poor capacitance tunability. MIM varactor shows higher tunability than IDC because the electric field primarily exists between the top and bottom electrodes, so the fringe field is reduced. However, the MIM varactor requires BST etching to reach the bottom electrode, whereas IDC is a planar structure and does not require BST etching. CPC can be made without BST etching, but the layout of CPC is not suitable for practical applications as it requires an additional large patch area to imbricate the central patch. Therefore, the main design requirement is to reduce the process steps (mainly the BST etching process) while achieving a high capacitance tunability similar to the MIM varactor. In this work, the proposed MIM varactor with a floating metal structure reduces the process steps while achieving high capacitance tunability and capacitance density. The room temperature on-wafer Sparameter measurements were performed to characterize the proposed varactor.

\section{VARACTOR DESIGN}

\section{A. MIM Varactor with floating metal}

The proposed MIM varactor with floating metal is shown in Fig. 1. The varactor structure consists of a coplanar waveguide line (CPW) loaded with the MIM varactor between the center conductor and ground plane. Here, a MIM capacitor is formed when the top metal and bottom metal have an overlap with BST dielectric between the top and bottom metals, In this case, the bottom electrode is a floating metal. The electric field caused by the control voltage is concentrated at the gap between the narrow center conductor and the ground plane and above the 
1. Bottom electrode (Pt with $\mathrm{Cr}$ adhesive layer) deposition using RF sputtering 2. Bottom electrode patterning

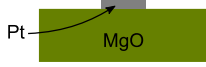

3. BST5 deposition using PLD

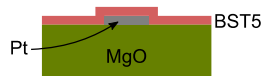

4. Top electrode (Au with $\mathrm{Cr}$ adhesive layer) deposition using RF sputtering 5. Top electrode patterning

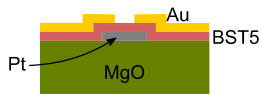

Fig. 2. Simplified flow chart of the fabrication process.

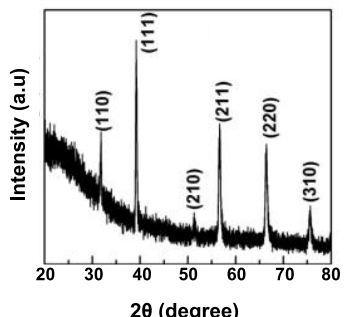

(a)

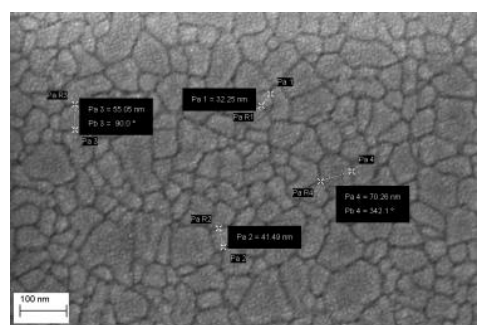

(b)
Fig. 3. XRD (a) and FESEM (b) image of the BST thin film on the MgO substrate.

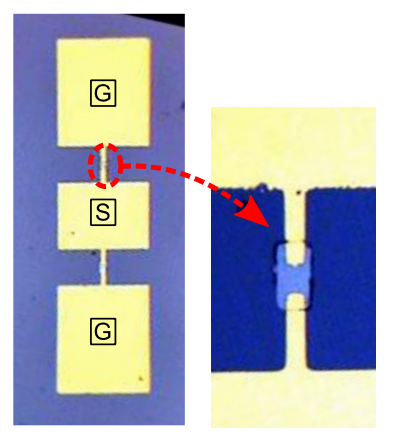

Fig. 4. Micrograph of the fabricated varactor structure on $\mathrm{MgO}$ substrate.

floating metal. This applied electric field changes the permittivity $\left(\varepsilon_{\mathrm{r}, \mathrm{BST}}\right)$ of the BST, thereby realizing the function of the varactor. As shown in Fig. 1, due to the floating metal, a series capacitance is formed between the center conductor and ground plane. In this case, each overlap presents a capacitance of $\mathrm{C}$, and the center conductor-to-ground plane capacitance on each side of the central conductor is $\mathrm{C} / 2$. Therefore, due to the CPW balanced structure, the total capacitance of the proposed varactor diode design is $\mathrm{C}$. The proposed varactor design does not require BST etching like that in prior MIM varactors, where BST etching requires additional process steps, and chemical processes during BST etching may reduce the quality of the film. Most importantly, the dielectric (BST) thickness is much lower than the area of the varactor, and a significant amount (almost complete) electric field is located inside the dielectric in the overlap region. Therefore, a high capacitance tunability can be achieved with the proposed varactor structure. In addition, the capacitance density of a capacitor depends on the $\left(\varepsilon_{\mathrm{r}, \mathrm{BST}} / \mathrm{d}\right)$ ratio. Therefore, due to the MIM structure with floating metal (reduced dielectric thickness d) and the high dielectric constant (300-500) of the BST film, can produce a higher capacitance density than IDC.

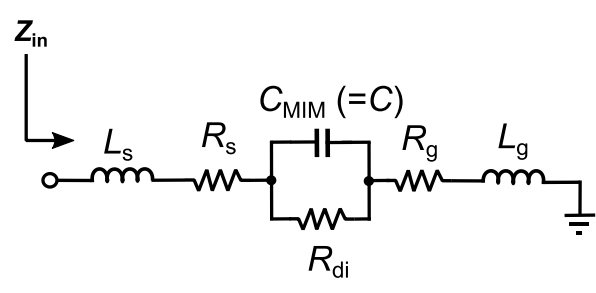

Fig. 5. Simplified equivalent circuit of the proposed varactor.

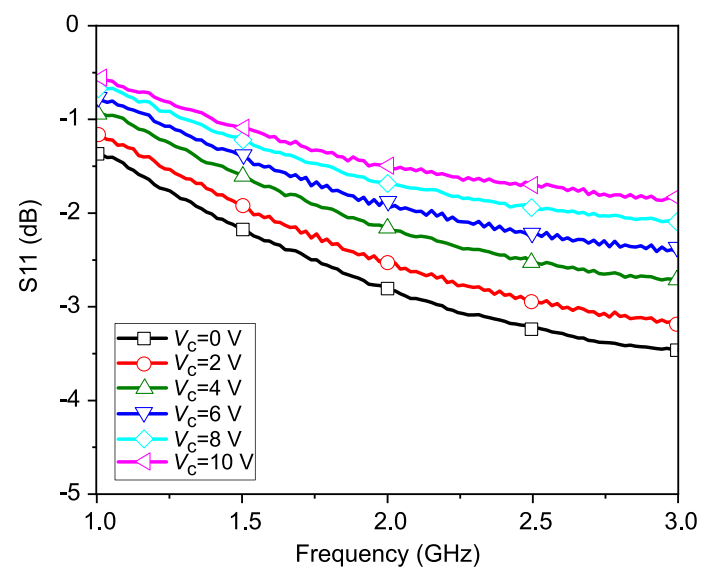

Fig. 6. Measured one port $S$-parameter results over control voltage $\left(V_{\mathrm{C}}\right)$.

In this case, the overlapping area of each MIM is $10 \times 10 \mu \mathrm{m}^{2}$. Since the capacitance is determined by the overlap area, by making the width of the bottom layer slightly larger than the width of the top metal, the capacitance change caused by small lithography alignment errors can be minimized. The gap between the center conductor and ground plane is optimized for a $50 \Omega$ line [14]. Fig. 5 presents the simplified equivalent circuit for the proposed varactor. The total capacitance of the varactor is given by $C_{\mathrm{MIM}}(=\mathrm{C})$, and $R_{\mathrm{di}}$ represents the leakage resistance in the dielectric. $L_{\mathrm{s}}, L_{\mathrm{g}} R_{\mathrm{s}}$, and $R_{\mathrm{g}}$ represent the center conductor and ground plane parasitic inductances and resistances. $R_{\mathrm{g}}$ and $R_{\mathrm{s}}$ originate due to metal thickness below the skin depth of the metals, resulting in skin resistance. Intuitively, from the equivalent circuit point of view, the high-frequency quality factor is affected by the $L_{\mathrm{g}}, L_{\mathrm{s}}, R_{\mathrm{g}}$, and $R_{\mathrm{s}}$.

\section{B. Fabrication}

The simplified fabrication process of the proposed varactor is shown in Fig. 2. At first, 100-nm platinum $(\mathrm{Pt})$ with chromium $(\mathrm{Cr})$ adhesive layer is deposited using RF sputtering for the bottom electrode and patterned using the lift-off process. After that, BST thin film is deposited using a pulsed laser deposition (PLD) system. The thin film quality is analyzed in measures of structural, microstructure properties, and surface thickness by using X-ray diffraction (GIXRD, Bruker D8 Discover) and Field emission scanning electron microscopy (FESEM, Carl Zeiss Ultra55). The deposition conditions are optimized for high-quality thin films (thickness 400-nm) on $\mathrm{MgO}$ substrate. As shown in Fig. 3, the BST thin film is strongly oriented towards (111) and highly polycrystalline. Since the BST thin film requires to be post-annealed at $750^{\circ} \mathrm{C}$, the choice of the bottom metal layer is very critical. In this work, platinum $(\mathrm{Pt})$ is used as the bottom metal due to its high temperature withstand capability. A standard photoresist (OiR 


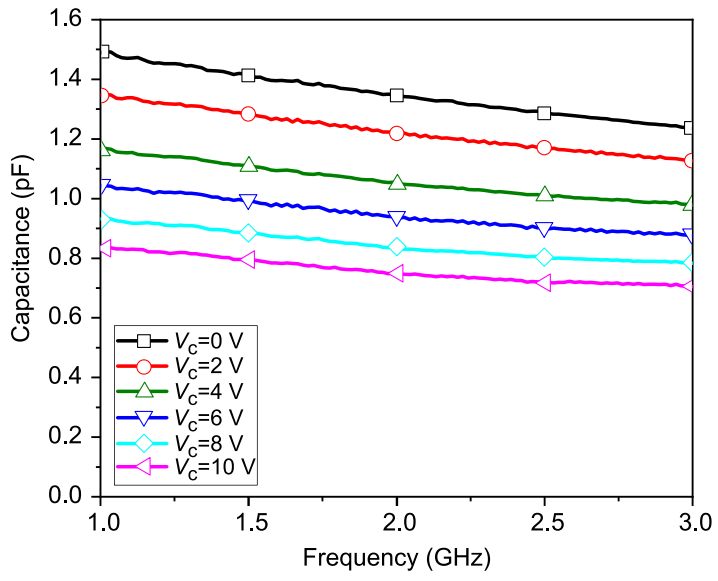

Fig. 7. Extracted varactor capacitance (C) from one port S-parameter results over control voltage $\left(V_{\mathrm{C}}\right)$.

620 ) is used to pattern top and bottom electrodes using the liftoff process. For the top electrode, 200-nm of gold (Au) layer is used along with 20-nm chromium (Cr) adhesive layer.

\section{MEASUREMENT Results}

Fig. 4 shows a photomicrograph of the fabricated varactor. One-port on-wafer S-parameter measurement was performed using a GSG probe $(250 \mu \mathrm{m})$ and VNA (Agilent E8361C) in the frequency range from $1 \mathrm{GHz}$ to $3 \mathrm{GHz}$ at room temperature. One-port open short-circuit load (OSL) calibration in the range of $0.5 \mathrm{GHz}$ to $10 \mathrm{GHz}$ was performed using GGB standard substrate (CS10) before the varactor S-parameter measurement. The control voltage $\left(V_{\mathrm{C}}\right)$ for the varactor structure is applied through a bias-tee. During the measurement, the control voltage of the varactor was varied from $0 \mathrm{~V}$ to $10 \mathrm{~V}$ in $2 \mathrm{~V}$ steps. Fig. 6 shows the measured $\mathrm{S}$-parameter results.

$$
\begin{gathered}
C=\frac{\left|\operatorname{Im}\left\{\left(1-S_{11}\right) /\left(1+S_{11}\right)\right\}\right|}{2 \pi f Z_{0}} \\
\text { Tunability }=\frac{C\left(V_{\mathrm{C}}=0 \mathrm{~V}\right)-C\left(V_{\mathrm{C}}=10 \mathrm{~V}\right)}{C\left(V_{\mathrm{C}}=0 \mathrm{~V}\right)} \times 100 \% \\
Q=\frac{\left|\operatorname{Im}\left\{Z_{\text {in }}\right\}\right|}{\operatorname{Re}\left\{Z_{\text {in }}\right\}}
\end{gathered}
$$

Where, $Z_{\text {in }}=Z_{0}\left(1+S_{11}\right) /\left(1-S_{11}\right)$

The capacitance $(C)$ of the fabricated varactor can be extracted using the eqn. (1) [15]. Among them, $f$ represents the frequency of interest, and $Z_{0}$ represents the characteristic impedance $(50 \Omega)$ of the GSG probe. The extracted capacitance over the control voltage of the varactor is shown in Fig. 7. As expected for BST materials, with the increase in control voltage, the value of the extracted capacitance decreases significantly. For $V_{\mathrm{C}}=10 \mathrm{~V}$, the capacitance changes from 1.49 $\mathrm{pF}$ to $0.83 \mathrm{pF}$ at $1 \mathrm{GHz}$, resulting in $44 \%$ tunability. The effective capacitance density at $1 \mathrm{GHz}$ and $V_{\mathrm{C}}=0 \mathrm{~V}$ is 3.7 $\mathrm{fF} / \mu \mathrm{m}^{2}$.The capacitance dispersion at higher frequencies is due to the increase of parasitic inductances at higher frequencies. As shown in Fig. 8, the $Q$-factor of the varactor is improved at a higher control voltage due to lower losses. In the entire frequency and bias voltage range, the capacitance change

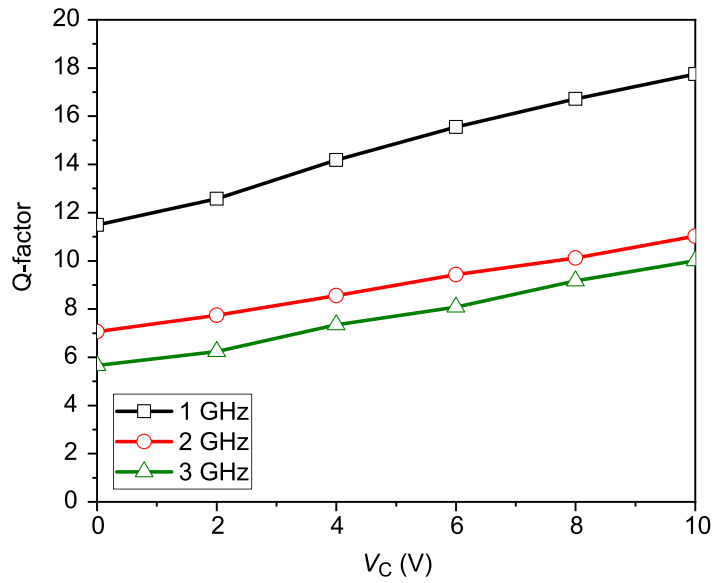

Fig. 8. Extracted $Q$-factor of the proposed varactor over control voltage $\left(V_{\mathrm{C}}\right)$.

TABLE I. COMPARISON TABLE OF PRIOR ART

\begin{tabular}{|c|c|c|c|c|c|c|}
\hline Ref. & {$[10]$} & {$[11]$} & {$[12]$} & {$[7]$} & {$[15]$} & This work \\
\hline Frequency (GHz) & 18 & 5 & 3.8 & 2 & 2.45 & 1 \\
\hline Capacitance (pF) @ 0V & 1.5 & 0.32 & 0.32 & 1.2 & 7.5 & 1.5 \\
\hline Tunability (\%) & 20 & 45 & 35 & 81 & 70 & 44 \\
\hline Control voltage (V) & $0-55$ & $0-30$ & $0-30$ & $0-20$ & $0-10$ & $0-10$ \\
\hline FoM & 2.5 & 10 & & 12 & 6 & 7.2 \\
\hline Varactor type & IDC & IDC & IDC & MIM & MIM & MIM \\
\hline BST etching & No & No & No & Yes & Yes & No \\
\hline
\end{tabular}

caused by process variation is within 5\%. The performance of the proposed varactor structure is further compared with other varactor structures, and Table I. lists the important matrices. The proposed varactor offers a low voltage and high capacitance tuning range for any varactor without BST etching. In this case, the drop in $Q$-factor is related to thin metal layers along with tracks and pads that are much larger than the effective (MIM overlap) area. As indicated in [7], a much thick metal layer $(1.5 \mu \mathrm{m})$ with an on-wafer de-embedding structure shows better FoM. These factors account for the reduced FoM but it is not because of the design.

\section{CONCLUSION}

BST shunt MIM varactor with a floating metal structure has been characterized on $\mathrm{MgO}$ substrate for the tunable coplanar waveguide circuits. The inclusion of floating metal effectively reduces the process steps of the MIM varactor while achieving a low voltage, high capacitance tunability, and capacitance density. The fabricated device shows $44 \%$ capacitance tunability over $1 \mathrm{GHz}$ to $3 \mathrm{GHz}$ for a control voltage of $0 \mathrm{~V}$ to $10 \mathrm{~V}$.

\section{REFERENCES}

[1] G. Jin, C. Deng, Y. Xu, J. Yang and S. Liao, "Differential FrequencyReconfigurable Antenna Based on Dipoles for Sub-6 GHz 5G and WLAN Applications," IEEE Antennas and Wireless Propag. Lett., vol. 19, no. 3, pp. 472-476, Mar. 2020.

[2] Y.I.A. Al-Yasir et al., "A Varactor-Based Very Compact Tunable Filter with Wide Tuning Range for 4G and Sub-6 GHz 5G Communications," MDPI Sensors, vol. 20, no. 16, pp. 4538, Aug. 2020.

[3] A. Anand, J. Small, D. Peroulis and X. Liu, "Theory and Design of Octave Tunable Filters With Lumped Tuning Elements," IEEE Trans. Microw. Theory Techn., vol. 61, no. 12, pp. 4353-4364, Dec. 2013. 
[4] C. Kong, H. Li, X. Chen, S. Jiang, J. Zhou and C. Chen, "A Monolithic AlGaN/GaN HEMT VCO Using BST Thin-Film Varactor," IEEE Trans. Microw. Theory Techn., vol. 60, no. 11, pp. 3413-3419, Nov. 2012.

[5] S. Preis, F. Lenze, A. Wiens, R. Jakoby, W. Heinrich and O. Bengtsson, "Reconfigurable packaged GaN power amplifier using thin-film BST varactors," in Proc. 47th Eur. Micrw. Conf. (EuMC), Oct. 2017, pp. 140143.

[6] K. Annam, D. Spatz, E. Shin and G. Subramanyam, "Experimental Verification of Microwave Phase Shifters Using Barium Strontium Titanate (BST) Varactors," in Proc. IEEE Nat. Aero. and Electr. Conf. (NAECON), Jul. 2019, pp. 63-66.

[7] R. De Paolis, S. Payan, M. Maglione, G. Guegan and F. Coccetti, "HighTunability and High-Q-Factor Integrated Ferroelectric Circuits up to Millimeter Waves," IEEE Trans. Microw. Theory Techn., vol. 63, no. 8, pp. 2570-2578, Aug. 2015.

[8] J. Nath et al., "An electronically tunable microstrip bandpass filter using thin-film Barium-Strontium-Titanate (BST) varactors," IEEE Trans. Microw. Theory Techn., vol. 53, no. 9, pp. 2707-2712, Sept. 2005.

[9] Guru Subramanyam et al., "Challenges and opportunities for multifunctional oxide thin films for voltage tunable radio frequency/microwave components," J. Appl. Phys., vol. 114, no. 19, pp. 191301, Nov. 2013.
[10] B. Ouagague et al., "BST tunability study at DC and microwave frequencies by using IDC and MIM capacitors," in Proc. Asia-Pacific Microw. Conf., Dec. 2010, pp. 1837-1840.

[11] A. Ghalem, F. Ponchel, D. Remiens, J. Legier and T. Lasri, "Broadband Dielectric Characterization of Sapphire/ $\mathrm{TiO}_{\mathrm{x}} / \mathrm{Ba}_{0.3} \mathrm{Sr}_{0.7} \mathrm{TiO}_{3}$ (111)Oriented Thin Films for the Realization of a Tunable Interdigitated Capacitor," IEEE Trans. Ultrason., Ferroelectr., Freq. Control, vol. 60, no. 5, pp. 880-887, May 2013.

[12] A. Ghalem, F. Ponchel, D. Remiens and T. Lasri, "A $3.8 \mathrm{GHz}$ tunable filter based on ferroelectric interdigitated capacitors," in Proc. IEEE Int. Symp. Applicat. Ferroelectr. (ISAF/PFM), July 2013, pp. 252-256.

[13] T.-G. Kim et al., "Crystallinity Dependence of Microwave Dielectric Properties in (Ba,Sr)TiO 3 Thin Films," Jpn. J. Appl. Phys., vol. 42, no. 3, pp. 1315-1319, Mar. 2003.

[14] Hang-Ting Lue and Tseung-Yuen Tseng, "Application of on-wafer TRL calibration on the measurement of microwave properties of $\mathrm{Ba}_{0.5} \mathrm{Sr}_{0.5} \mathrm{TiO}_{3}$ films," IEEE Trans. Ultrason., Ferroelectr., Freq. Control, vol. 48, no. 6, pp. 1640-1647, Nov. 2001.

[15] A. Ghalem et al., "Ultra-High Tunability of $\mathrm{Ba}_{(2 / 3)} \operatorname{Sr}_{(1 / 3)} \mathrm{TiO}_{3}$-Based Capacitors Under Low Electric Fields," IEEE Microw. Wireless Compon. Lett., vol. 26, no. 7, pp. 504-506, Jul. 2016. 